\title{
Asymmetry in the periodicities of solar photospheric fields: A probe to the unusual solar minimum prior to cycle 24
}

\author{
Susanta Kumar Bisoi and P. Janardhan
}

Physical Research Laboratory, Astronomy \& Astrophysics Division, Ahmedabad 380 009, India email: susanta@prl.res.in

\begin{abstract}
Employing wavelet and Fourier methods, we investigate temporal variations of periodicities in the photospheric fields obtained from synoptic magnetograms of the National Solar Observatory at Kitt Peak (NSO/KP) spanning the years 1975-2009. A north-south asymmetry is noticed in the periodicities of photospheric fields in the latitude range, $45^{\circ}-78^{\circ}$, when the data is grouped into fields prior to and after 1996. This asymmetry when coupled with the fact that both solar fields in the latitude range, $45^{\circ}-78^{\circ}$, and the micro-turbulence levels in the inner heliosphere began declining $\sim 1995-1996$ suggests that active changes occurred in the underlying basic solar processes which eventually initiated, at the end of solar cycle 23 , the build-up of the deepest solar minimum, in the past 100 years.
\end{abstract}

Keywords. Sun: magnetic field, Sun: periodicity, and Sun: interior.

\section{Introduction}

Investigation of periodicities in solar photospheric fields reveals the connection between the evolution of these fields and the underlying basic processes in the solar interior. Recently, prior to the start of solar cycle 24, we have experienced one of the deepest solar minimum in the past 100 years. It has been reported by Janardhan et al., 2011 that the build-up to this deep solar minimum had started over a decade earlier, based on the steady decline they found in the solar polar fields and the micro-turbulence levels in the inner heliosphere since $\sim 1995-1996$. In this work, we attempt to investigate the role of periodicity changes of photospheric fields in the buildup to this solar minimum.

\section{Result and Implications}

The magnetic fields, obtained from synoptic maps of the NSO/KP database in the years 1975-2009, are smoothed by Savitzky \& Golay algorithm for estimating magnetic residuals. These magnetic residuals are further subjected to a wavelet and Fourier analysis for unevenly spaced data. Figure 1 depicts the result obtained from these two methods for the fields in the higher latitude ranging from $45^{\circ}$ to $78^{\circ}$. The top two panels show the wavelet power distribution of periodicities for these high-latitude fields in the north and south respectively. It is clear that the resultant wavelet power distribution show a transition in periodicity since mid-1990's.

For the Fourier analysis, we have divided the data set into fields before and after 1996, with 1996 being the year where the wavelet spectra showed a sharp change. The bottom four panels in pairs show the normalized Fourier power of periodicities for the north and south in the period prior to 1996 and after 1996. It is evident that an hemispheric asymmetry exists and shown as the shift in the peaks of highest periodic components that are in the opposite direction for the north and south as indicated by the solid lines, 

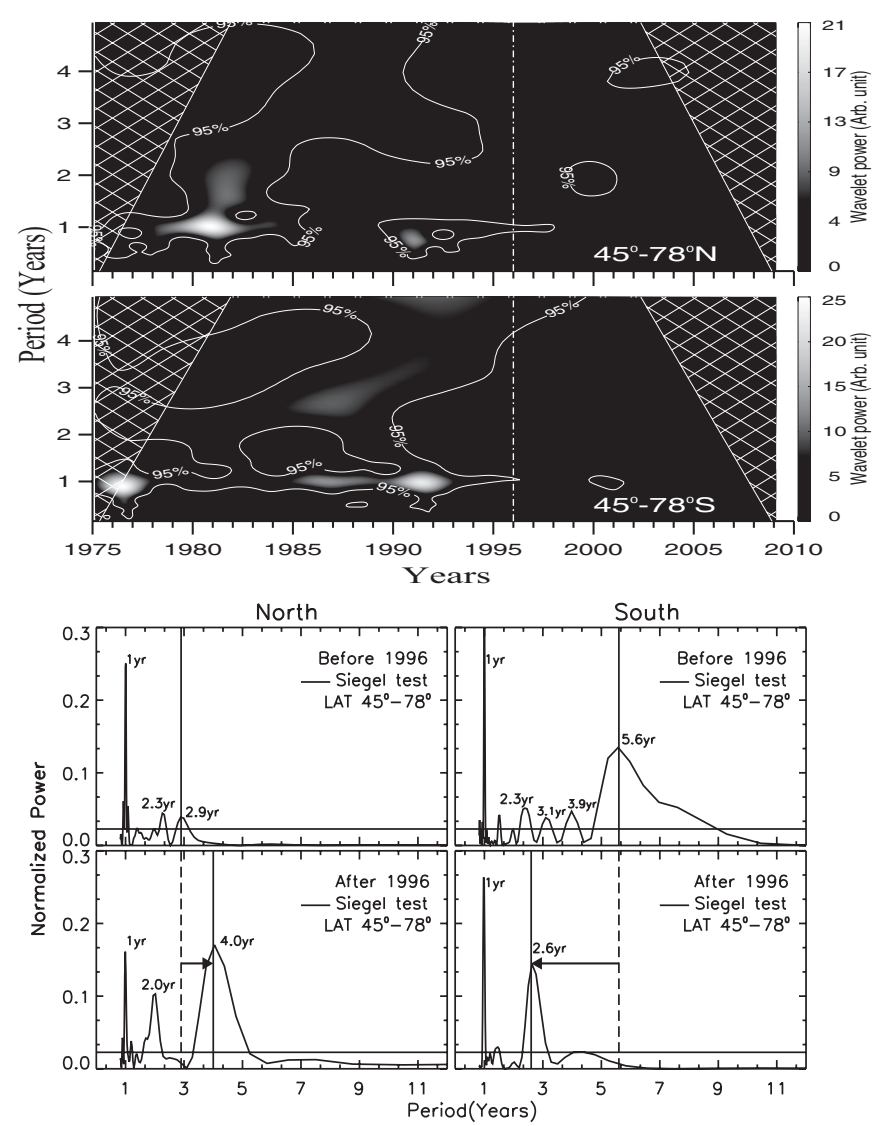

Figure 1. The top two panels show the wavelet power distribution of periodicities of photospheric fields for the north (upper panel) and the south (lower panel) in the latitudes ranging from $45^{\circ}$ to $78^{\circ}$. The white cross hatched regions are the cone of influence while the white contours indicate siginficance level at $95 \%$. The vertical dotted lines mark the period of transition in wavelet powers $\sim 1996$. The lower four panels represent the normalized fourier power of periodicities for high-latitude fields. The two panels in pairs at left and at right respectivley depict Fourier power for the north and south prior to 1996 and after 1996. The solid lines, the dotted lines and the arrows in the Figure indicate the shift in the peaks of longest periodic component while the horizontal solid lines are marked at significance levels as determined by Siegel's test.

dotted lines and the arrows. Since high-latitude fluxes primarily depend on the poleward motions of fluxes that vary with active region eruptions and surface meridional flows rates, so any changes in these fields can be attributed to changes in the solar interior. Thus, this asymmetry coupled with the declining solar polar fields and micro-turbulence levels in the inner heliosphere 1995 -1996 suggests active changes occurred inside the sun and those, in turn, initiated the buildup to the deep solar minimum at the end of the cycle 23 .

\section{References}

Janardhan, P., Bisoi, S. K., Ananthakrishnan, S., Tokumaru, M., \& Fujiki, K. 2011, Geophys. Res. Lett., 382, 20108 